\title{
Mathematical modeling of a small pressure disturbance in gas flow of a long pipeline
}

\author{
Chekurin V. ${ }^{1}$, Khymko O. ${ }^{2}$ \\ ${ }^{1}$ Pidstryhach Institute for Applied Problems of Mechanics and Mathematics \\ National Academy of Sciences of Ukraine \\ 3-b Naukova str., 79060, Lviv, Ukraine \\ ${ }^{2}$ Lviv Polytechnic National University \\ 12 S. Bandera str., 79013, Lviv, Ukraine
}

(Received 10 December 2017)

\begin{abstract}
A mathematical model for propagation of a small disturbance in the moving gas of a long pipeline has been built in the paper. The model contains two coupled equations, the coefficients of which are expressed via the parameters of the initial process - mass density and mass flow rate. In the frame of the model, the influence of parameters of the initial stationary gas flow on propagation of the pulses initiated by a local fluctuation, originated in the stream, was studied numerically. The developed model can be used for quantitative analysis of oscillations of operational regimes of the long-distance pipeline, which are caused by random stream fluctuations and/or operational pulsation of the compressors. The model can be also useful to study negative pressure waves emerging in the pipeline during its local depressurization.
\end{abstract}

Keywords: nonlinear problems, non-stationary processes, gas dynamics, gas flow in long pipeline, wave in moving gas, small disturbance propagation.

2000 MSC: 35Q35, 65M06

UDC: $534.13,519.6$

DOI: $10.23939 / \mathrm{mmc} 2017.02 .126$

\section{Introduction}

Gas main pipelines are giant engineering structures, containing of buried and ground-surface pipelines of a big diameter (up to $1.4 \mathrm{~m}$ ). The length of a pipeline section between two compressor stations can reach $100-150 \mathrm{~km}$. The main pipelines are effectively used for long distance transportation of natural gas. Their high productivity can be achieved by creating of high pressures by compressor stations at the sections inlets. The pressure decays downstream the section because of the friction between the moving gas and pipe wall, and varies depending on the pipeline's path profile. Therefore the metal of pipe body is in inhomogeneous strain-stressed state produced by the friction and intrinsic pressure. Modes of operation, which is determined by the flow rate and inlet pressure of a pipeline, can change cyclically on day, week, month and season bases. Cyclic stress of the metal in combination with other destructive processes (corrosion etc.) can provoke its cracking with consequent pipeline depressurization.

Pressure and flow rate values are usually monitored at the inlets and outlets of each section and often at several points along its route. These data are used for operational control of the pipeline. They can be used also for detection of unpredictable pipeline depressurization, leakage location and its identification $[1,2]$. In this connection, we can mention two known methods of leak detection in gas pipelines.

The first one is so called method of negative pressure waves [1-3]. The idea of the method is that the sharp pressure jump, emergent at the depressurization point, produces two waves of negative pressure

This work was supported by grants of NASU, State registration 0113 U007687 and 0116 U007435. 
drop, one of which propagates downstream, another one - upstream. Measuring time moments $t_{1}$ and $t_{2}$ of the wave arrival at the points $x_{1}$ and $x_{2}$, one of which is situated downstream, and another one upstream of the point of depressurization, one can calculate the coordinate $x_{0}$ of the depressurization point if flow rate is known.

Another method, called Real Time Transient Modeling (RTTM) $[1,2,4]$ is based on numerical solving of non-stationary boundary value problems of gas dynamics, which model mass, momentum and energy transfer in the pipeline. The functions of boundary conditions for these problems are being obtained on the base of measurements of pressure and flow rate values at the section input and output. Measuring additionally pressure and flow rate values at several points along the path of the section and juxtapose the obtained data to numerical solutions of the boundary value problems, one can judge about leak presence, its location and intensity. There are several modifications of RTTM method, differing in ways of acquisition and initial processing of the empirical data, and by algorithms of their utilization [4].

As we can see, both methods are based on precise measuring of the pressure and flow rate values and their variations, caused by the depressurization. But waves of pressure and flow arise in the pipeline gas stream not only because of the depressurization. Operational pulsation of the compressors, turbulent instability of the flow caused by local hydraulic resistances, random fluctuations of pressure, temperature, density and composition of transported gas, etc. can also be reason of emerging of wave disturbances of pressure and flow rate of moving gas in the pipeline. Experimental data presented in paper [5] show that the amplitude of pressure pulsation measured in a pipeline equals about $1 \%$ of operational pressure value. These local disturbances create an acoustic noise for registration of the negative pressure drop waves and variations of pressure and flow rate caused by local depressurization, what can reduce sensitivity and precision of the above mentioned methods for leaks detection and location. Therefore, studying the behavior of small disturbances in the gas flow of main pipelines is a problem of great scientific and practical importance.

The paper is devoted to mathematical modeling and numerical study of small acoustic disturbances in the moving gas of long-distance pipelines.

\section{Governing equations}

We consider a section of a main gas-pipeline as a long cylindrical pipe of the length $L$ and the constant diameter $D$. Let a natural gas of known composition is transported via the section. Motion of the gas in the pipe is governed by the equations of mass, momentum and energy transfer. In the present paper, we restrict our consideration to an isothermal approach, in which the model includes two conservation equations of mass and momentum [6]:

$$
\begin{gathered}
\frac{\partial \rho}{\partial t}+\frac{\partial \rho V}{\partial x}=0 \\
\frac{\partial \rho V}{\partial t}+\frac{\partial}{\partial x}(P+\rho V V)+\frac{\lambda}{2 D} \rho V|V|+g \frac{\partial h}{\partial x} \rho=0 .
\end{gathered}
$$

Here $\rho, P$ and $V$ stand for the mass density of the gas, its pressure and velocity being functions of the coordinate $x$ and time $t, h=h(x)$ is the pipeline's axis elevation, depending on the coordinate $x$ along the pipeline trace, $\lambda$ stands for friction coefficients, $g$ is gravitational acceleration.

As the equations (1) and (2) contain three unknown functions, we will consider them simultaneously with a thermal equation of state, which connects dependent variable $P(x, t)$ and $\rho(x, t)$, and can be written in two equivalent forms $[6,7]$ :

$$
P=z R_{g} \rho T \quad \text { or } \quad \rho=\frac{P}{z R_{g} T} .
$$


We used here the denotations: $R_{g} \equiv R / M_{g}$, where $R$ is universal gas constant, $M_{g}$ is the gas molar mass; $z$ stands for gas compressibility factor, it defines deviations of thermodynamic properties of real gas from the model of ideal gas, for which $z=1$.

There are various analytical approximations, obtained with the use of empirical data, which consider $z$ as a function of pressure $P$ and temperature $T$ (at a fixed gas composition). In such presentation formula $(3)_{1}$ gives an implicit representation of pressure $P$ as a function of density $\rho$ and temperature $T$. Of course one can solve the equation $(3)_{1}$ with respect to $P$ and thus determine the compressibility factor $z$ as a function of density $\rho$ and temperature $T$. Further we will consider equations (3) in explicit form, supposing parameter $z$ in the first equation (3) is known as the function $z=z(\rho, T)$, but in the second one - as $z=z(P, T)$.

Equations (1)-(3) form a system for determination variables $\rho, P$ and $V$ as function of coordinate $x$ and time $t$. Knowing them one can determine mass flow density $J=\rho V$ and volumetric flow rate $Q=J \pi D^{2} /\left(4 \rho_{s t}\right)$, where $\rho_{s t}$ stands for the value of gas density at the standard conditions.

A regime of section operation is determined by dependences of pressure $P$ and mass flow density $J=\rho V$ on coordinate $x$ and time $t$. It established by the pressures $P_{i n}, P_{\text {out }}$ and/or by mass flows $J_{\text {in }}, J_{\text {out }}$ values creating by the compressor stations at the inlet and outlet of the section. According to this we can consider, four pair of boundary conditions:

$$
\begin{aligned}
\left.P\right|_{x=0} & =P_{\text {in }}(t), & \left.P\right|_{x=L} & =P_{\text {out }}(t) . \\
\left.P\right|_{x=0} & =P_{\text {in }}(t), & \left.J\right|_{x=L} & =J_{\text {out }}(t) . \\
\left.J\right|_{x=0} & =J_{\text {in }}(t), & \left.P\right|_{x=L} & =P_{\text {out }}(t) . \\
\left.J\right|_{x=0} & =J_{\text {in }}(t), & \left.J\right|_{x=L} & =J_{\text {out }}(t) .
\end{aligned}
$$

Here $P_{\text {in }}(t), P_{\text {out }}(t), J_{\text {in }}(t), J_{\text {out }}(t)$ are given function, which can be determined, in particular, empirically by measuring pressure $P$ and mass flow density $J$ at the section inlet and outlet.

To formulate a problem for system the pair of initial conditions

$$
\left.P\right|_{t=0}=P_{\text {init }}(t),\left.\quad J\right|_{t=0}=J_{\text {init }}(t),
$$

should be also taken into consideration.

We assume the boundary conditions (4)-(7) are consistent to the initial conditions (8), i.e.:

$$
P_{\text {in }}(0)=P_{\text {init }}(0), \quad P_{\text {out }}(0)=P_{\text {init }}(L), \quad J_{\text {in }}(0)=J_{\text {init }}(0), \quad J_{\text {out }}(0)=J_{\text {init }}(L) .
$$

Eliminating pressure $P$ from equation (2) with the use of relation (3), we obtain the system of partial differential equations for determination of density $\rho$ and mass flow $J=\rho V$ in the form

$$
\begin{gathered}
\frac{\partial \rho}{\partial t}+\frac{\partial J}{\partial x}=0 . \\
\frac{\partial J}{\partial t}+\left(a^{2}-\frac{J^{2}}{\rho^{2}}\right) \frac{\partial \rho}{\partial x}+2 \frac{J}{\rho} \frac{\partial J}{\partial x}+\frac{\lambda}{2 D} \frac{J|J|}{\rho}+g \frac{\partial h}{\partial x} \rho=0
\end{gathered}
$$

where $a=a(\rho)$ is acoustic speed:

$$
a^{2}=\partial P / \partial \rho=(z+\rho \partial z / \partial \rho) R_{g} T
$$

Taking $\rho(x, t)$ and $J(x, t)$ as the key functions, we obtained the system of governing equations (10), (11), the first of which is linear one. We reduce the system to a dimensionless form. For that we use typical values for pressure $P_{t}$ and mass flow density $J_{t}$. Calculating the typical value of the mass density by formula $(3)_{2}: \rho_{t}=P_{t} /\left(z\left(P_{t}, T\right)\right)$, and time period $t_{t} \equiv L / a_{t}$, where $L$ is the length of the pipeline, $a_{t}$ stands for acoustic speed at $\rho=\rho_{t}$ : $a_{t}=\sqrt{\left(z\left(\rho_{t}, T\right)+\rho_{t} \partial z /\left.\partial \rho\right|_{\rho=\rho_{t}}\right) R_{g} T}$, we introduce 
dimensionless mass density $\bar{\rho} \equiv \rho / \rho_{t}$, mass flow density $j \equiv J / J_{t}$, dimensionless time $\tau \equiv t / t_{t}$ and coordinate $\xi \equiv x / L$. Finally, we obtain governing equations in the dimensionless form [8]

$$
\begin{gathered}
\frac{\partial \bar{\rho}}{\partial \tau}+M a \frac{\partial j}{\partial \xi}=0, \\
\frac{\partial j}{\partial \tau}+\frac{1}{M a}\left(\bar{a}^{2}-M a^{2} \frac{j^{2}}{\bar{\rho}^{2}}\right) \frac{\partial \bar{\rho}}{\partial \xi}+2 M a \frac{j}{\bar{\rho}} \frac{\partial j}{\partial \xi}+\beta M a \frac{j|j|}{\bar{\rho}}+M a \frac{\partial \bar{h}}{\partial \xi} \omega=0,
\end{gathered}
$$

where

$$
\bar{a} \equiv \frac{a}{a_{t}}=\bar{a}(\xi, \tau), \quad M a \equiv \frac{V_{t}}{a_{t}}, \quad \beta \equiv \frac{\lambda L}{2 D}, \quad \bar{h} \equiv \frac{g}{V_{t}^{2}} h .
$$

The boundary (4)-(7) and initial (8) conditions for dimensionless variables take the forms:

$$
\begin{aligned}
& \left.\bar{\rho}\right|_{\xi=0}=\bar{\rho}_{i n}(\tau), \\
& \left.\bar{\rho}\right|_{\xi=1}=\bar{\rho}_{\text {out }}(\tau) . \\
& \left.\bar{\rho}\right|_{\xi=0}=\bar{\rho}_{i n}(\tau), \\
& \left.j\right|_{\xi=1}=j_{\text {out }}(t) \text {. } \\
& \left.j\right|_{\xi=0}=j_{\text {in }}(t), \\
& \left.\bar{\rho}\right|_{\xi=1}=\bar{\rho}_{\text {out }}(\tau) \text {. } \\
& \left.j\right|_{\xi=0}=j_{\text {in }}(t) \\
& \left.j\right|_{\xi=1}=j_{\text {out }}(t) \text {. } \\
& \left.\bar{\rho}\right|_{\tau=0}=\bar{\rho}_{\text {init }}(\xi), \\
& \left.j\right|_{\tau=0}=j_{\text {init }}(\xi) .
\end{aligned}
$$

Here

$$
\begin{aligned}
\rho_{\text {in }}(\tau) & =\frac{P_{\text {in }}\left(\tau \cdot t_{t}\right)}{z\left(P_{\text {in }}\left(\tau \cdot t_{t}\right), T\right) R_{g} T}, & \rho_{\text {out }}(\tau) & =\frac{P_{\text {out }}\left(\tau \cdot t_{t}\right)}{z\left(P_{\text {out }}\left(\tau \cdot t_{t}\right), T\right) R_{g} T}, \\
j_{\text {in }}(\tau) & =\frac{J_{\text {in }}\left(\tau \cdot t_{t}\right)}{J_{t}}, & j_{\text {out }}(\tau) & =\frac{J_{\text {out }}\left(\tau \cdot t_{t}\right)}{J_{t}}, \\
\rho_{\text {init }}(\xi) & =\frac{P_{\text {init }}(\xi \cdot L)}{z\left(P_{\text {init }}(\xi \cdot L), T\right) R_{g} T}, & j_{\text {init }}(\xi) & =\frac{J_{\text {init }}(\xi \cdot L)}{J_{t}} .
\end{aligned}
$$

In a stationary regime $\partial \bar{\rho} / \partial \tau=\partial j / \partial \tau \equiv 0$. It follows from (12) in this case that $\partial j / \partial \xi \equiv 0$, and we can reduce equation (13) to the form

$$
\frac{d \bar{\rho}}{d \bar{\xi}}=-M a^{2} \frac{\beta \bar{\rho} j^{2}+\frac{\partial \bar{h}}{\partial \xi} \bar{\rho}^{3}}{\left(\bar{a}^{2} \bar{\rho}^{2}-M a^{2} j^{2}\right)}
$$

In this case we can subordinate the function $\bar{\rho}(\xi)$ to one of the next boundary conditions

$$
\left.\bar{\rho}\right|_{\xi=-1}=\bar{\rho}_{\text {in }},\left.\quad \bar{\rho}\right|_{\xi=1}=\bar{\rho}_{\text {out }},
$$

where $\bar{\rho}_{\text {in }}$ and $\bar{\rho}_{\text {out }}$ are given constants.

\section{Mathematical model for small disturbances}

Let consider a gas pipeline in an operational state, determined by the pair of functions $\bar{\rho}_{0}(\xi, \tau)$ and $j_{0}(\xi, \tau)$. Let a small disturbance $\tilde{\rho}(\xi, \tau)$ and $\tilde{j}(\xi, \tau)$ has emerged in the gas stream. Then the disturbed state is defined by functions

$$
\bar{\rho}(\xi, \tau)=\bar{\rho}_{0}(\xi, \tau)+\tilde{\rho}(\xi, \tau), \quad j(\xi, \tau)=j_{0}(\xi, \tau)+\tilde{j}(\xi, \tau) .
$$

The pairs $\bar{\rho}_{0}(\xi, \tau), j_{0}(\xi, \tau)$ and $\bar{\rho}(\xi, \tau), j(\xi, \tau)$ both satisfy the equations (13), (14). Started from this and taking into account the smallness of the disturbance $\tilde{\rho}(\xi, \tau), \tilde{j}(\xi, \tau)$, we will establish governing equations for functions $\tilde{\rho}(\xi, \tau), \tilde{j}(\xi, \tau)$. 
As (13) is a linear equation, the disturbance $\tilde{\rho}(\xi, \tau), \tilde{j}(\xi, \tau)$ satisfies its exactly:

$$
\frac{\partial \tilde{\rho}}{\partial \tau}+M a \frac{\partial \tilde{j}}{\partial \xi}=0
$$

Substituting (23) into (14) and neglecting by terms, which are nonlinear with respect to the functions $\tilde{\rho}, \tilde{j}$ and their derivatives, we obtain

$$
\begin{aligned}
\frac{\partial \tilde{j}}{\partial \tau}+\frac{1}{M a}\left(\bar{a}_{0}^{2}-M a_{0}^{2}\right) \frac{\partial \tilde{\rho}}{\partial \xi}+2 M a_{0} \frac{\partial \tilde{j}}{\partial \xi}+2 & \left(\frac{\partial M a_{0}}{\partial \xi}+\beta M a_{0}\right) \tilde{j} \\
& +\frac{1}{M a}\left(\frac{\partial \bar{a}_{0}^{2}}{\partial \xi}-\frac{\partial M a_{0}^{2}}{\partial \xi}-\beta M a_{0}^{2}+M a^{2} \frac{d \bar{h}}{d \xi}\right) \tilde{\rho}=0
\end{aligned}
$$

where $M a_{0} \equiv M a \cdot v_{0}=\frac{V_{t}}{a_{t}} \frac{V_{0}}{V_{t}}=\frac{V_{0}}{a_{t}}=M a_{0}(\xi, \tau)$.

The coefficients of the linearized equation (25) are defined in terms of parameter of non-disturbed gas flow $\bar{\rho}_{0}(\xi, \tau)$ and $v_{0}(\xi, \tau)=j_{0}(\xi, \tau) / \bar{\rho}_{0}(\xi, \tau)$. In common case they are functions of spatial coordinate $\xi$ and time $\tau$ and can be calculated on the base of the solution of system (13), (14), subordinated to one of boundary conditions (16)-(20).

In the case of stationary initial state the coefficients of (25) do not depend on time variable $\tau$ and are determined through density $\bar{\rho}_{0}(\xi)$, which, in turn, can be defined by solving ordinary differential equation (21):

$$
\frac{d \bar{\rho}_{0}}{d \xi}=-M a^{2} \frac{\beta \bar{\rho}_{0} j_{0}^{2}+\frac{\partial \bar{h}}{\partial \xi} \bar{\rho}_{0}^{3}}{\bar{a}_{0}^{2} \bar{\rho}_{0}^{2}-M a^{2} j_{0}^{2}},
$$

at given $j_{0}=$ const and one of the boundary conditions (22).

Since the coefficients of equation (25) in this case do not depend on time variable $\tau$, we can reduce the system (24), (25) to one governing equation:

$$
\begin{aligned}
\frac{\partial^{2} \tilde{j}}{\partial \tau^{2}}-\left(\bar{a}_{0}^{2}-M a_{0}^{2}\right) \frac{\partial^{2} \tilde{j}}{\partial \xi^{2}}+2 M a_{0} \frac{\partial^{2} \tilde{j}}{\partial \xi \partial \tau}+2 & \left(\frac{d M a_{0}}{d \xi}+\beta M a_{0}\right) \frac{\partial \tilde{j}}{\partial \tau} \\
& -\left(\frac{d \bar{a}_{0}^{2}}{d \xi}-\frac{d M a_{0}^{2}}{d \xi}-\beta M a_{0}^{2}\right) \frac{\partial \tilde{j}}{\partial \xi}-M a^{2} \frac{d \bar{h}}{d \xi} \frac{\partial \tilde{j}}{\partial \xi}=0 .
\end{aligned}
$$

Consider this equation in some particular cases.

In the case of horizontal $(\partial h / \partial x=0)$ blanked-off pipeline $\left(J=0\right.$ and $\left.P=P_{t}\right)$, we have $a_{0}=1$, $M a_{0}=0$. In this case equation (27) transforms to the form of conventional wave equation:

$$
\frac{\partial^{2} \tilde{j}}{\partial \tau^{2}}-\frac{\partial^{2} \tilde{j}}{\partial \xi^{2}}=0
$$

which admits solutions in forms of the plane waves, one of which propagates in the positive direction and another - in the negative direction of axis:

$$
\tilde{j}(x, t)=F_{1}(\xi+\tau)+F_{2}(x-t) .
$$

Here $F_{1}(\ldots)$ and $F_{2}(\ldots)$ are functions, which should be determined from the initial conditions.

The disturbance $\tilde{\rho}$ as the function of coordinate $\xi$ and time $\tau$ can be found from equation (24) with the use of (29):

$$
\tilde{\rho}(x, t)=-M a\left(F_{1}(\xi+\tau)-F_{2}(x-t)\right) .
$$

As the dimensionless friction coefficient $\beta$ is proportional to the pipeline's length $L$, it decreases when $L$ diminishes. Hence, a case of rather short pipeline the friction coefficient $\beta$ be small enough, 
and we can put it equal to zero. In this case, in a horizontal pipeline, as it follows from equation (26), the gradient $\partial \bar{\rho}_{0} / \partial \xi$ of dimensionless density in the initial state is also small: $\partial \bar{\rho}_{0} / \partial \xi=\mathrm{o}(\beta)$. So, we can ignore the spatial inhomogeneity of mass density and suppose $\bar{a}_{0}=$ const, $M a_{0}=$ const. This reduces equation $(27)$ to the form

$$
\frac{\partial^{2} \tilde{j}}{\partial \tau^{2}}-\left(\bar{a}_{0}^{2}-M a_{0}^{2}\right) \frac{\partial^{2} \tilde{j}}{\partial \xi^{2}}+2 M a_{0} \frac{\partial^{2} \tilde{j}}{\partial \xi \partial \tau}=0
$$

The general solution of this equation can be presented as the superposition of two plane waves propagating in opposite directions of the axis $\xi$ with different phase velocities $\bar{a}_{0} \pm M a_{0}$ :

$$
\tilde{j}(x, t)=F_{1}\left(\left(\bar{a}_{0}-M a_{0}\right) \tau+\xi\right)+F_{2}\left(\left(\bar{a}_{0}+M a_{0}\right) \tau-\xi\right) .
$$

The disturbance $\tilde{\rho}$ as the function of coordinate $\xi$ and time $\tau$ can be found by integration of the equation (24) with the use of the solution (32).

\section{Coefficients of the governing equation}

To study numerically how stationary initial state influences the coefficients of governing equation (27), we considered a horizontal pipeline with friction coefficient $\lambda=0.0092$, diameter $1.338 \mathrm{~m}$ and length $120 \mathrm{~km}$ and chose typical values for pressure $P_{t}=6.65 \mathrm{MPa}$ and flow rate $J_{t}=483.95 \mathrm{~kg} / \mathrm{m}^{2} \mathrm{~s}$. On this basis, supposing gas temperature $T=300 \mathrm{~K}$, we calculated the typical mass density $\rho_{t}=50.52 \mathrm{~kg} / \mathrm{m}^{3}$, flow velocity $V_{t} \equiv J_{t} / \rho_{t}=9.5798 \mathrm{~m} / \mathrm{s}$, sound velocity $a_{t}=337.62 \mathrm{~m} / \mathrm{s}$, time period $t_{t}=355.43 \mathrm{~s}$. Mach number $M a \equiv V_{t} / a_{t}=0.0284$, and friction coefficient $\beta=413.46$.

We determine the initial stationary regime by values of the pressure $P_{i n}$ at the inlet of the pipeline and mass flow rate $J_{0}$. Corresponding dimensionless variables are $\bar{\rho}_{i n}$ and $j_{0}$. To calculate the coefficients of the governing equation as functions of coordinate $\xi$, we solve the initial Cauchy problem for the nonlinear differential equation (26) with the initial condition $(22)_{1}:\left.\bar{\rho}_{0}\right|_{\xi=-1}=\bar{\rho}_{i n}$. Using the solution $\bar{\rho}_{0}=\bar{\rho}_{0}(\xi)$, we calculate the parameters $\bar{a}^{2}=\bar{a}^{2}(\xi)$ and $M a_{0}^{2}=M a_{0}^{2}(\xi)$

$$
\bar{a}^{2}=\left(z\left(\rho_{t} \bar{\rho}_{0}, T\right)+\rho_{t} \bar{\rho}_{0} \partial z /\left.\partial \rho\right|_{\rho=\rho_{t} \bar{\rho}_{0}}\right) R_{g} T / a_{t}^{2}, \quad M a_{0}^{2}=M a^{2} j_{0}^{2} / \bar{\rho}_{0}^{2} .
$$

In Fig. 1, we can see how dimensionless density $\bar{\rho}_{0}$ and normalized sound speed $\bar{a}_{0}$ are dependent on coordinate $\xi$ along the pipeline (plots $a$ and $b$ correspondingly). The calculations, results of which are presented in Fig. 1 , were made at value $\bar{\rho}_{i n}=1$ for various values of the dimensionless mass flow density $j_{0}=0.6,0.8$, and 0.9 (curves 1,2 , and 3 correspondingly).

We call a stationary regime, determining by the pair $\left(\bar{\rho}_{i n}, j_{0}\right)$, as critical if the solution $\bar{\rho}_{0}(\xi)$ of initial-value problem for the equation (26) with the initial condition $\left.\bar{\rho}_{0}\right|_{\xi=-1}=\bar{\rho}_{\text {in }}$ satisfies the condition $\left.\bar{\rho}_{0}\right|_{\xi=1}=0$, i.e. if the value of mass density (and pressure) at the outlet becomes zero. At $\bar{\rho}_{i n}=1$, the critical value of dimensionless mass flow density $j_{0}$ lays between 0.9068 and 0.9069 . Hence the regime determined by the pair $\left(\bar{\rho}_{i n}, j_{0}\right)=(1.0,0.9)$ is very close to critical.

As we see, the variability of the functions $\bar{\rho}_{0}(\xi)$ and $\bar{a}_{0}(\xi)$ increases with increasing $j_{0}$. The acoustic speed is less variable - it varies in the range about $15 \%$ at the greatest studied value of the flow density $j_{0}=0.9$. At $j_{0}=0.6$, the mass density varies in the range about $15 \%$, whereas the sound velocity changes its values in the range about several percents. At $j_{0}=0.9$, the mass density (and pressure) value at the outlet is several times less against it value at the inlets.

The parameters $\bar{a}_{0}^{2}$ and $M a_{0}^{2}$ are constituents of the second coefficient of the equation (28). The figure 2 illustrates how they change along the pipeline. The curves 1,2 , and 3 in this figure were calculated for different values of dimensionless mass flow $j_{0}=0.6,0.8$, and 0.9 . The left vertical axis in Fig. $2 b$ refers to the curves 1 and 2 , the right one - to the curve 3. 

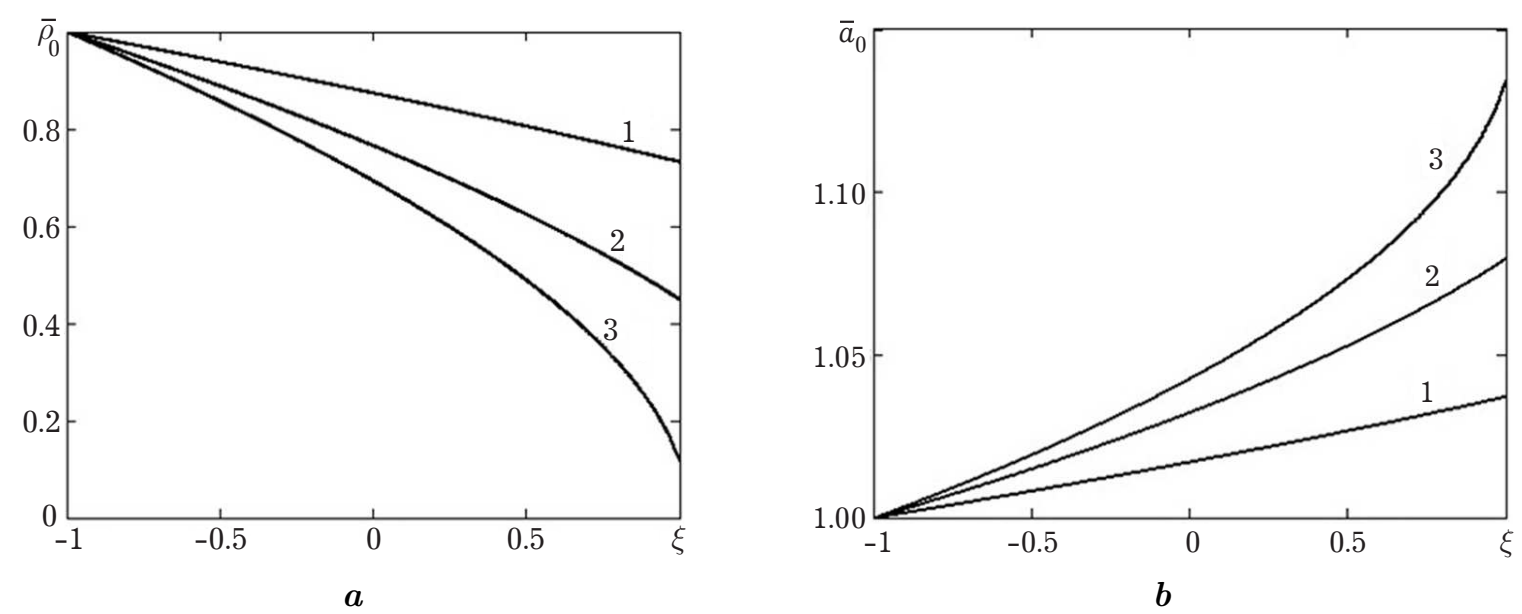

Fig. 1. Coordinate dependences of mass density $(a)$ and acoustic speed $(b)$ of initial stationary regime at different values of mass flow.
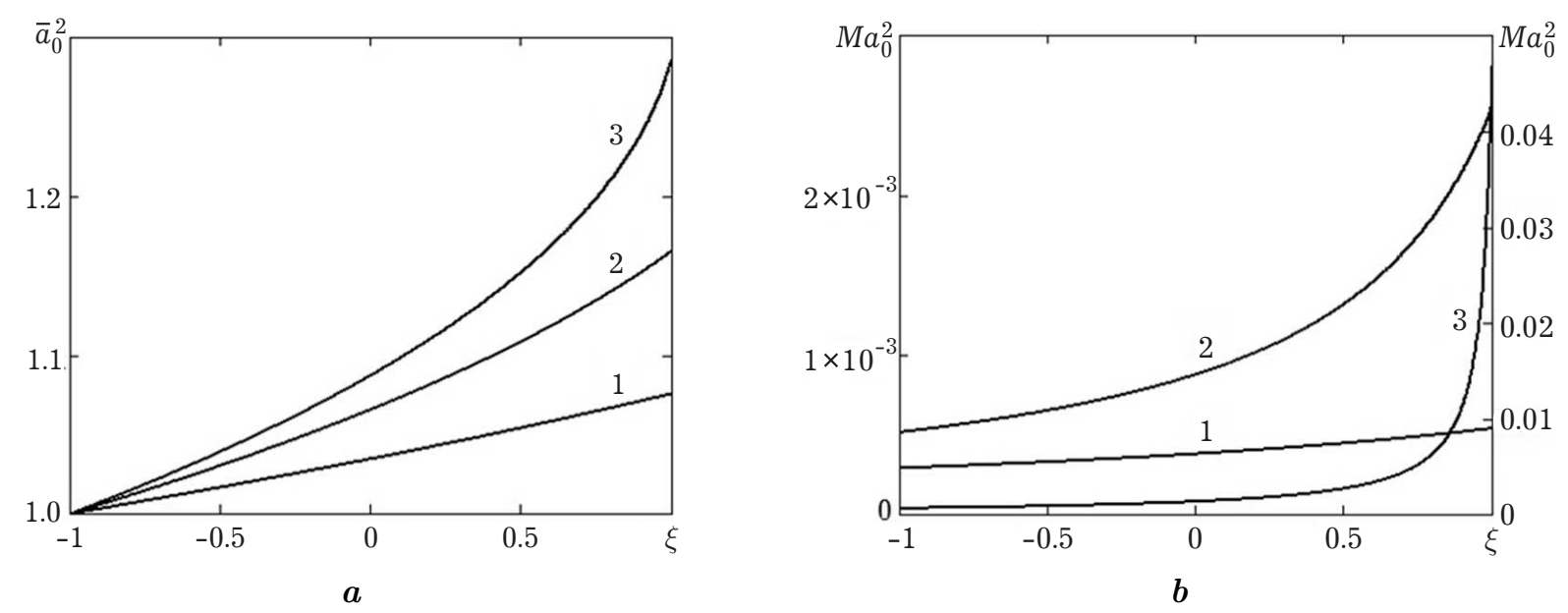

Fig. 2. Coordinate dependences of the constituents of the second coefficient of Eq. (27).

As we see, the term $M a_{0}^{2}$ is small against $\bar{a}_{0}^{2}$ - it affects the values of the second coefficient only in its third or second decimal digit (depending on the value of mass flow).

The curves in Fig. $2 b$ give an idea of the variability of the third coefficient of the equation (28).

The forth coefficient of the equation (27) is dependent on the parameters $d M a_{0} / d \xi=M a \cdot d v_{0} / d \xi$ and $\beta \cdot M a_{0}=\beta \cdot M a \cdot v_{0}$. To calculate the first of them, we determine the derivative $\partial v_{0} / \partial \xi$ in term of solution $\bar{\rho}_{0}=\bar{\rho}_{0}(\xi)$ of the equation (17). Using the equation (16), we obtain

$$
\frac{d v_{0}}{d \xi}=\frac{M a^{2} \beta j_{0}^{3}}{\bar{a}_{0}^{2} \bar{\rho}_{0}^{3}-M a^{2} j_{0}^{2} \bar{\rho}_{0}}
$$

Analyzing (35), we can conclude that $\partial M a_{0} / \partial \xi$ is a quantity of order $M a^{3} \cdot \beta$, whereas $\beta \cdot M a_{0}=$ $\beta \cdot M a \cdot v_{0}$ is a quantity of order $\beta \cdot M a$. Since $\beta$ is much greater than the unity and $M a=\mathrm{o}(1)$, the parameter $\partial M a_{0} / \partial \xi$ is much less than the parameter $\beta \cdot M a_{0}$.

The figure 3 shows how the constituents of the fourth coefficient vary along the pipeline. Here the left vertical axis refers to the curves 1 and 2 , the left one - to the curves 3 .

Examining the plots, we can verify the validity of the above conclusion about the ratio of the values $\partial M a_{0} / \partial \xi$ and $\beta \cdot M a_{0}$. 

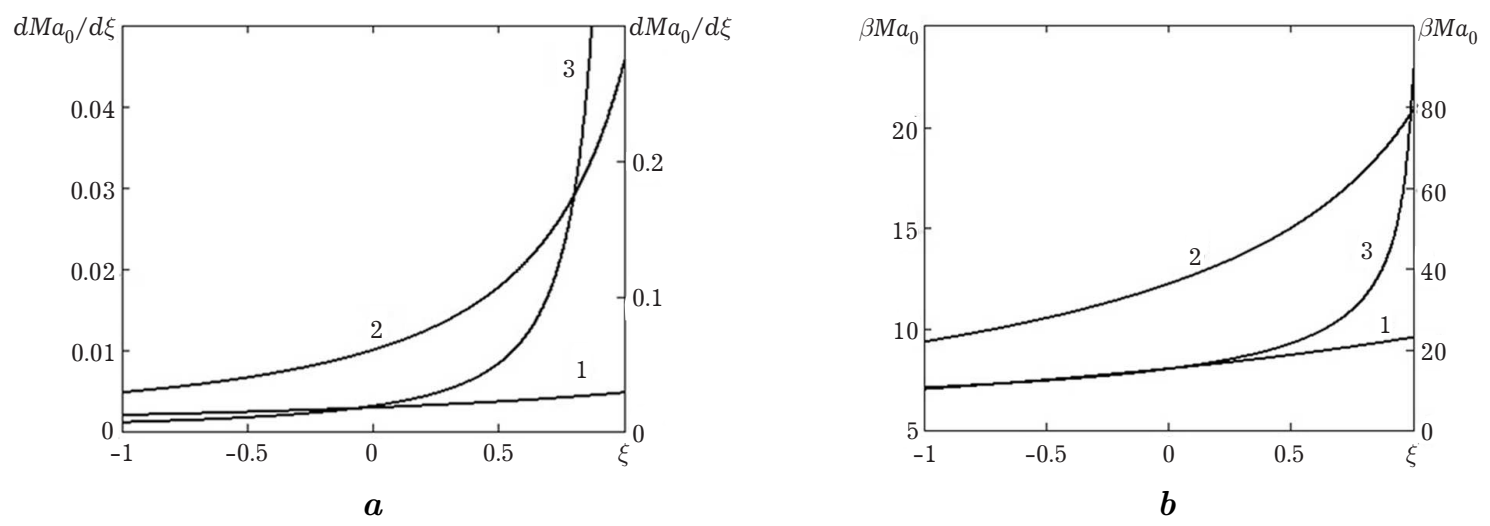

Fig. 3. Coordinate dependences of the constituents of the fourth coefficient of Eq. (27).

We determine the derivative $d \bar{a}_{0}^{2} / d \xi=\partial \bar{a}^{2} /\left.\partial \bar{\rho}\right|_{\bar{\rho}_{0}} d \bar{\rho}_{0} / d \xi$ with the use of the relation (12) and the equation (26):

$$
\frac{d \bar{a}_{0}^{2}}{d \xi}=-\frac{1}{a_{t}}\left(\left.2 \frac{\partial z}{\partial \rho}\right|_{\rho=\rho_{t} \bar{\rho}_{0}}+\left.\rho_{t} \bar{\rho}_{0} \frac{\partial^{2} z}{\partial \rho}\right|_{\rho=\rho_{t} \bar{\rho}_{0}}\right) \frac{M a^{2} \beta \bar{\rho}_{0} j_{0}^{2}}{\bar{a}_{0}^{2} \bar{\rho}_{0}^{2}-M a^{2} j_{0}^{2}} .
$$

To find the derivative $\partial M a_{0}^{2} / \partial \xi$, we express $\partial v_{0}^{2} / \partial \xi \equiv 2 v_{0} \partial v_{0} / \partial \xi$ in terms of mass density $\bar{\rho}_{0}$. Using the equation (35), we obtain

$$
\frac{d v_{0}^{2}}{d \xi}=\frac{2 M a^{2} \beta j_{0}^{4}}{\bar{a}_{0}^{2} \bar{\rho}_{0}^{4}-M a^{2} j_{0}^{2} \bar{\rho}_{0}^{2}}
$$
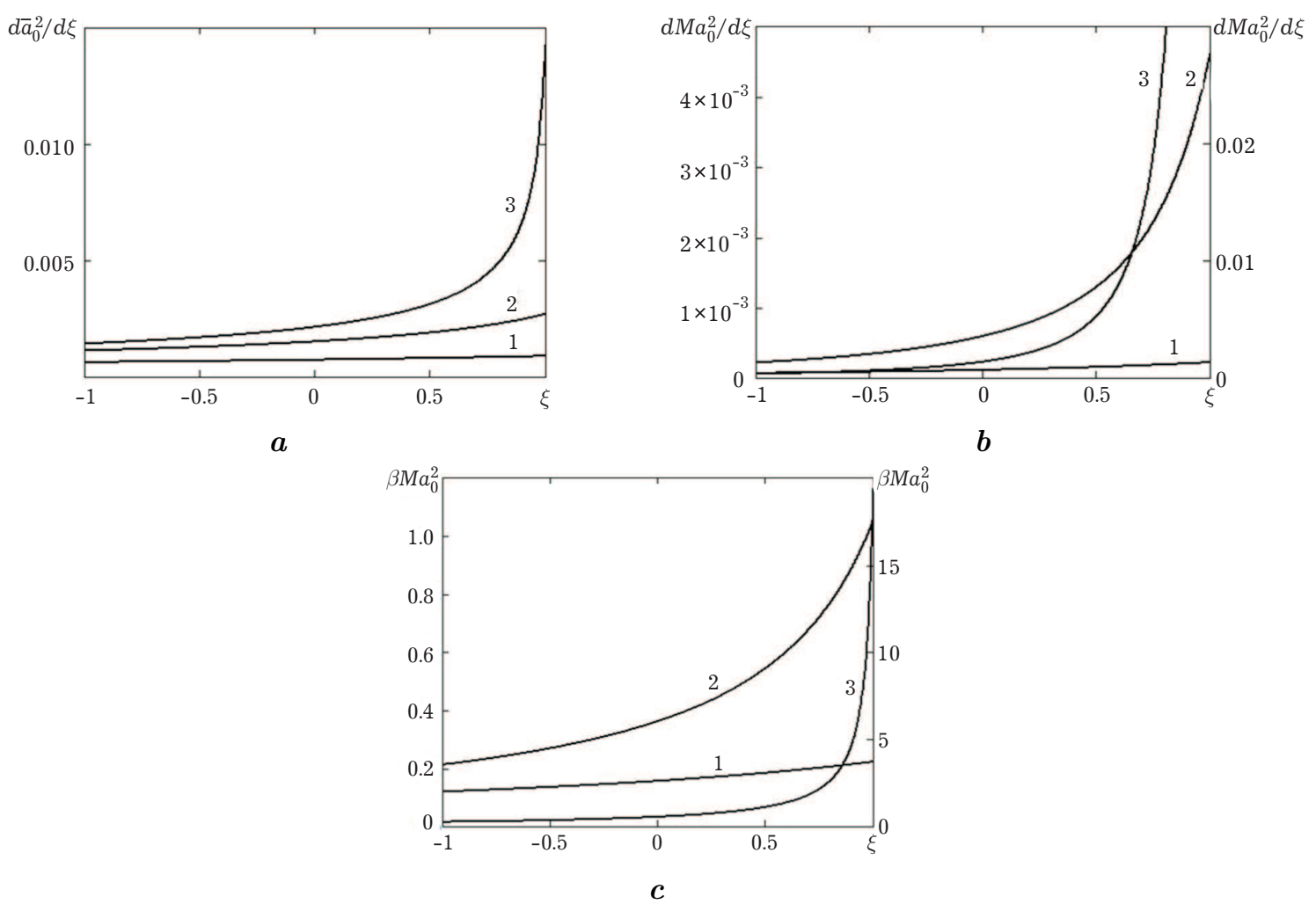

Fig. 4. Coordinate dependences of the constituents of the fifth coefficient of Eq. (27). 
On this basis, using the solution of the initial-value problem, determining the initial stationary regime, we can calculate the constituents of the fifth coefficient of the equation (27) as functions of the coordinate $\xi$. The results are presented in Fig. 4 . They were obtained at $\bar{\rho}_{i n}=1$ and different values of mass flow rate $j_{0}=0.6,0.8,0.9$ (curves $1,2,3$ correspondingly). The curves 2 and 3 in Figs. $4 b$ and $4 c$ refer to the left vertical axis, the curves 3 - to the right one.

It is follows from the graphs that the variability of the constituents of the fifth coefficient depends on the value of mass flow density: at constant $\bar{\rho}_{i n}$ - it rises with increasing of mass flow rate. The constituents $d \bar{a}_{0}^{2} / d \xi$ and $\partial M a_{0}^{2} / \partial \xi$ are small parameters as compared with $\beta \cdot M a_{0}^{2}$.

\section{Damping of fluctuations in the moving gas}

Let us consider the problem of attenuation of the local density fluctuation $\tilde{\rho}=\tilde{\rho}(\xi, \tau)$, which arose in the moving gas during the initial stationary regime. The regime is determined by the inlet density $\bar{\rho}_{\text {in }}$ : $\left.\bar{\rho}_{0}\right|_{\xi-1}=\bar{\rho}_{i n}$ and the mass flow rate density $j_{0}$. We model the fluctuation by initial conditions, taking them in the form

$$
\left.\tilde{\rho}\right|_{\tau=0}=\varphi(\xi),\left.\quad \tilde{j}\right|_{\tau=0}=0,
$$

where $\varphi(\xi)$ is a given function.

We suppose the disturbance does not affect the mode of pipeline operation imposing the homogeneous boundary conditions:

$$
\left.\tilde{\rho}\right|_{\xi= \pm 1}=0 .
$$

The fluctuation disturbs the initial stationary flow, but we suppose the deviations of the density $\tilde{\rho}(\xi, \tau)$ and flow rate $\tilde{j}(\xi, \tau)$ are small as compared to initial stationary parameters $\bar{\rho}_{0}$ and $j_{0}$. It enables us to use the above developed model to study disturbance propagation in the moving gas. To do this we solved the problem (24), (25), (37), (38) numerically with the use finite difference method. We considered a local disturbance, which has arose in a small vicinity of a point $\xi=\xi_{0} \in(-1,1)$. For this we putted in $(37)_{1} \varphi(\xi)=f\left(\xi-\xi_{0}\right)$, where $f(\xi)$ is a single pulse taken in the form

$$
f(x)= \begin{cases}\cos \frac{\pi \xi}{2 d}, & |\xi| \leqslant d \\ 0, & d<|\xi| \leqslant 1\end{cases}
$$

Here $d>0$ defines the width of the pulse.

As the problem is linear, we normalize the function $\tilde{\rho}=\tilde{\rho}(\xi, \tau)$ on its maximal value at the initial time moment $\tau=0$.

Some results of conducted numerical studies are presented in Figs. 5 and 6 . The plots in the figures illustrate how mass (Fig. 5) and flow rate (Fig. 6) densities of the fluctuation decay in the pipeline depending on the point of its originating and the value of initial mass flow rate $j_{0}$. Positions (a), (c) and (e) of the figures refer to $j_{0}=0.6$, position (b), (d) and (f) refer to $j_{0}=0.9$. Plots on the positions (a) and (b), (c) and (d), (e) and (f) on the both figures were calculated for different points of the fluctuation origination correspondingly. Curves numbers 1 to 6 correspond the dimensionless time moments $0.08,0.13,0.20,0.33$.

Analyzing the plots, we can see that behavior of the density fluctuation depends on the place of its origination and on the initial (undisturbed) flow rate.

It can be explained by peculiarities of coordinate dependences of the coefficients of the equation (27), particularly - the terms $\beta \cdot M a_{0}$ and $\beta \cdot M a_{0}^{2}$ in fourth and fifth coefficients correspondingly. These terms are dominant in their coefficients, which determine the dissipativity of the system. As show the plots in Figs. 4 and 5 the dissipativity of system rapidly increases with increasing of the initial flow rate $j_{0}$ and with approaching to the pipeline outlet $\xi=1$. Inhomogeneity of the coefficients also increases at that. 


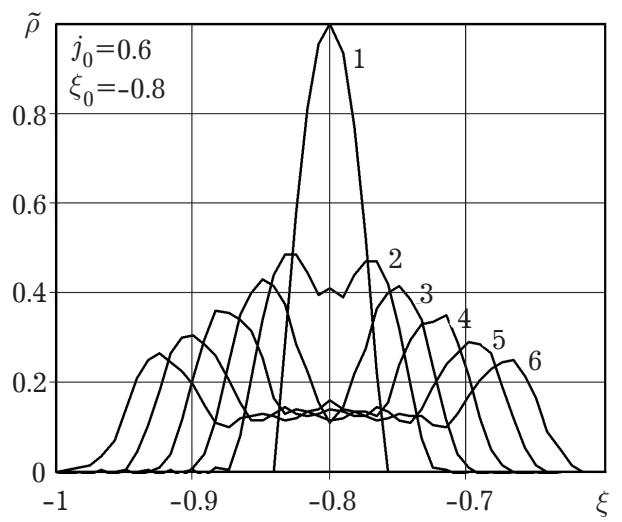

a

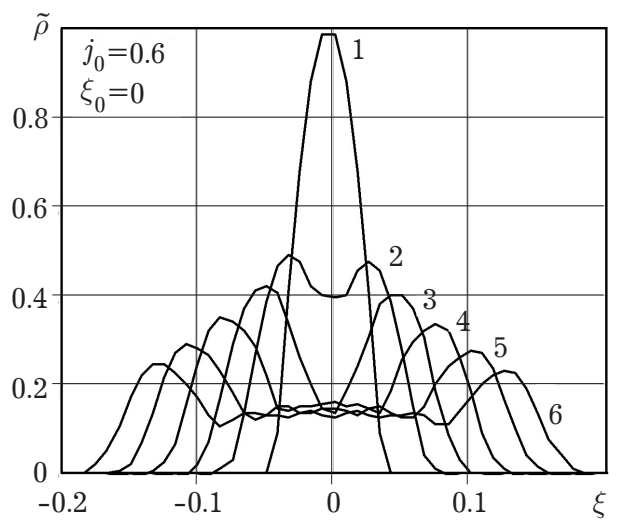

$c$

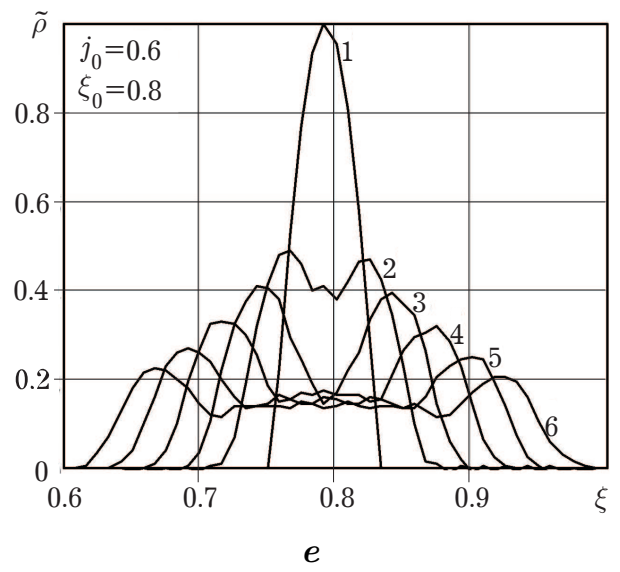

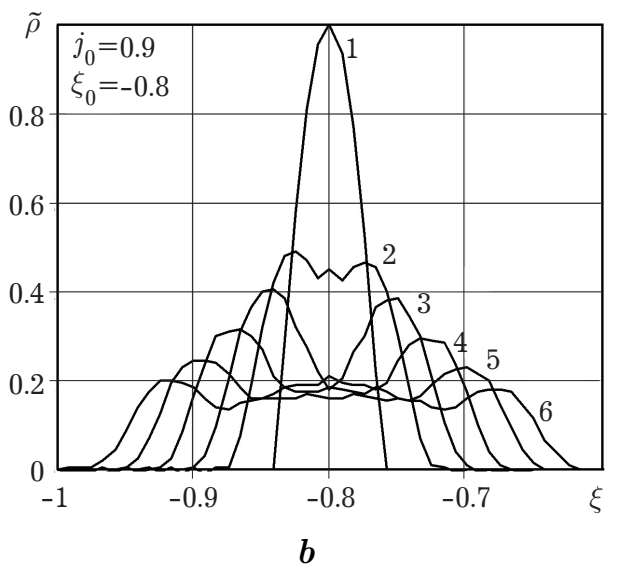
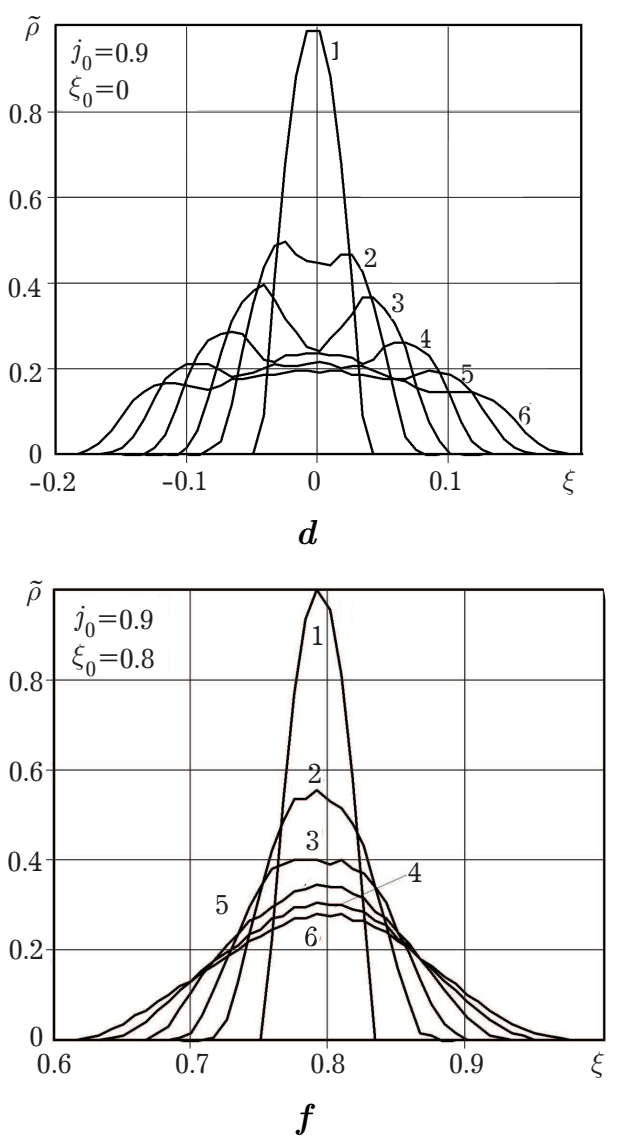

Fig. 5. Coordinate dependences of mass density of local fluctuations originating in vicinities of various points $\xi_{0}$ for two different values of the initial mass flow rate $j_{0}$ and fixed value of initial inlet density $j_{i n}=1$.

At low initial flow rate $\left(j_{0}=0.6\right)$ the disturbance originated close to the inlet $\left(\xi_{0}=-0.8\right)$ possesses typical features of a wave process with attenuation (see Figs. $5 a, 6 a$ ). In this domain of the pipeline, the flow velocity, dissipativity and inhomogeneity are rather small. That is why dying down pulses, propagating upstream and downstream of the flow from the point of fluctuation originating, are practically identical.

Looking at the curves of Fig. $5 c$, we can observe some asymmetry of the field pattern - the pulses propagating downstream attenuate faster than those propagating upstream. It is because the flow velocity and inhomogeneity of the coefficients near-by the midpoint of the pipeline are greater than near the inlet. A stronger asymmetry of the field pattern is observed in vicinity of the point $\xi_{0}=0.8$ (Figs. $5 e$ and $6 e$ ). 

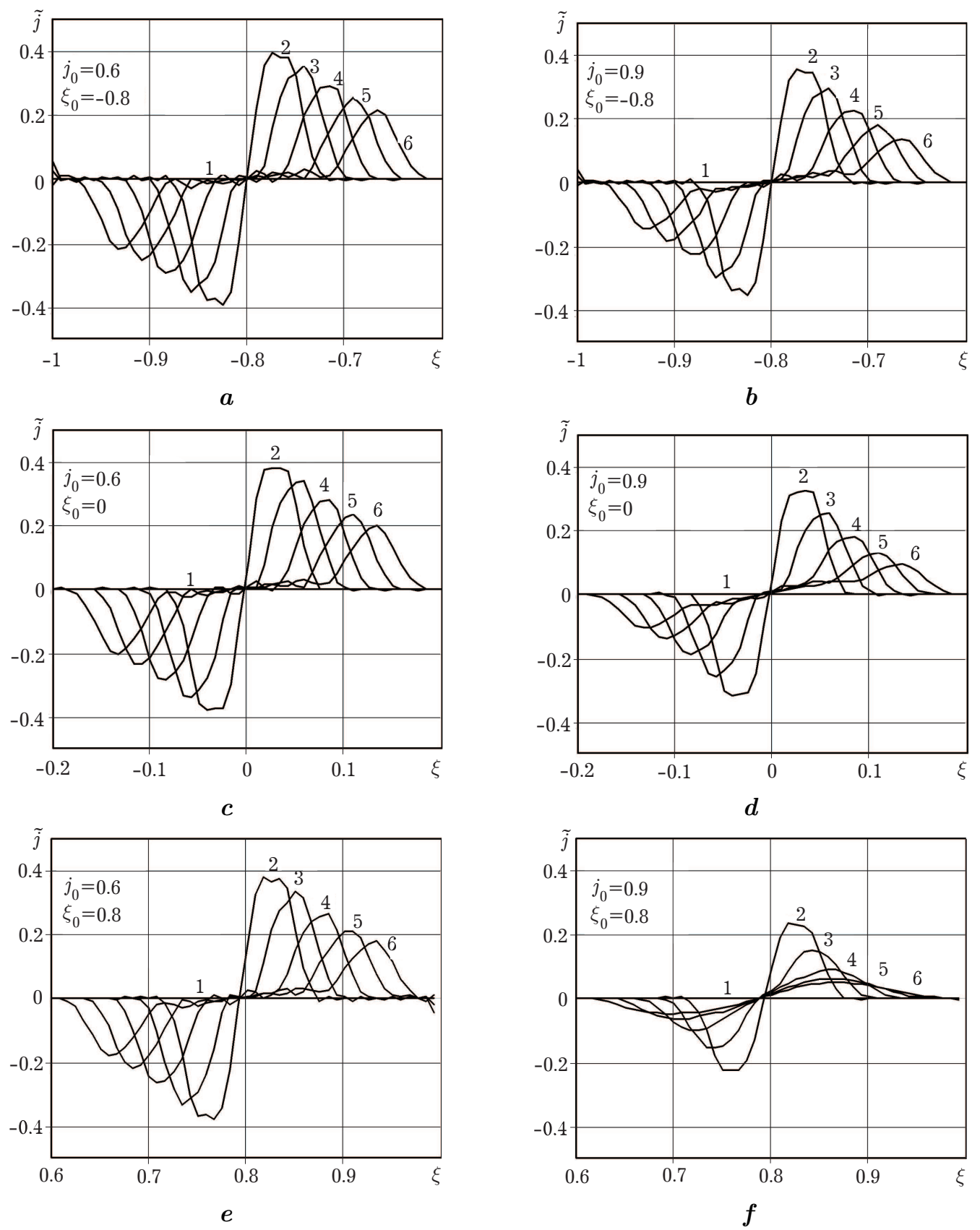

Fig. 6. Coordinate dependences of mass flow rate density of local fluctuations originating in vicinities of various points $\xi_{0}$ at two different values of the initial mass flow rate $j_{0}$ and fixed value of initial inlet density $j_{\text {in }}=1$.

The asymmetry of wave pattern goes up with increasing of initial flow rate from $j_{0}=0.6$ to $j_{0}=0.9$. We can observe this in Figs. $5 b, 6 b$ and $5 d, 6 d$ where the pulses, initiated by fluctuations, originated near-by the inlet and at the middle point of the pipeline, are shown. As we can observe, the pulses, propagating down- and upstream from the origination points, demonstrate similar behavior typical for wave process with attenuation. But the asymmetry in these cases is stronger than at $j_{0}=0.6$. As the initial stationary regime $\left(\bar{\rho}_{i n}=1, j_{0}=0.9\right)$ is very close to critical, the coefficients and their gradients increase drastically near-by the outlet of the pipeline. This radically changes the field pattern (see fig. $5 f)$ - it losses the features typical for wave process and obtain the features typical for diffusive process. 


\section{Conclusions}

Vibrations of pressure and flow rate in operational regimes of main pipelines emerge because of pulsation of the compressors operation, variation of gas flow parameters, caused by the turbulence occurring near-by local hydraulic resistances, random fluctuations of temperature, etc. The effect of these fluctuations on the efficiency of gas transportation is not so important, but they reduce the sensitivity of known leak detection methods based on recording pressure pulses that occur when the pipeline is depressurized.

A linearized mathematical model for propagation of a small disturbance of pressure and flow rate in the moving gas of a long pipeline has been developed. It describes small deviations of field parameters from a given initial unsteady operational regime. The model is based on the nonlinear one-dimensional model of gas dynamics in the isothermal approximation. It includes two coupled equations, the coefficients of which are dependent on the field parameters of the initial (undisturbed) regime. As the parameters of initial regime are known, one can calculate the coefficients as functions of the coordinate and time. Using the developed model, one can formulate boundary-value problems for study of origination, propagation and decaying of small disturbances in the moving gas depending on the parameters of initial regime.

In the case of stationary initial processes, the coefficients are dependent only on the coordinate. For this case the developed model was reduced to one governing equation of hyperbolic type. Under certain assumptions about the initial stationary process, the equation can be reduced to forms that allow solutions in the form of a traveling wave.

The coefficients of obtained hyperbolic equation were studied numerically depending on the parameters of initial stationary process. It was established that the values of the coefficients and their gradients increase with approaching to the outlet of the pipeline and with rising of the flow rate of the initial stationary regime.

In the frame of the model the problem of propagation and decaying of the disturbance initiated by a local fluctuation of pressure was formulated. The numerical solution obtained with the use of finiteelement method, enabled to study features of the wave fields, initiated by local fluctuations emerging in various zones of the pipeline - near-by its inlet, at the midpoint of the pipeline and near-by its outlet. The influence of the value of the mass flow rate of initial stationary regime on the wave field was also studied. It was established, in particular, that the pattern of the wave field can radically change when the initial regime is close to the critical.

The developed model and obtained results of numerical study can be used to quantitative analysis of oscillations of operational regimes of the long-distance pipeline, which are caused by random stream fluctuations and/or operational pulsation of the compressors. The model can be also useful to numerical study negative pressure waves, emerging in the pipeline during its local depressurization. It can be used under development of mathematical tools and algorithms for methods of leak detection.

[1] Hamilton S., Charalambous H. Leack Detection: Technologies and Implementation. London, IWA Publishing (2013).

[2] Murvay P.-S., Silea I. A Survey on Gas Leak Detection and Localization Techniques. Journal of Loss Prevention in the Process Industry. 25 (6), 966-973 (2012).

[3] Hou Q., Ren L., Jiao W., Zou P., Song G. An Improved Negative Pressure Wave Method for. Natural Gas Pipeline Leak Location Using FBG Based Strain. Sensor and Wavelet. Mathematical Problems in Engineering. 2013, Article ID 278794, 8 pages (2013).

[4] Geiger G., Werner T, Matko D. Knowledge based Leak Monitoring for Pipelines. 4th IFAC Workshop on On-Line Fault Identification and Supervision in the Chemical Process Industries, June 7-8 2001, Jejudo Islands (Korea). 34 (27), 249-254 (2001). 
[5] Kjell-Edvind Froysa, Per Lunde, Anfinn Paulsen and Endre Jacobsen. Density and Caloric Value Measurement in NATUREL gas Using Ultrasonic Flow Meters. Results from Testing on Various North Sea Gas Field Data. 24th International North Sea Flow Measurement Workshop, 24th-27th October 2006.

[6] Shapiro A. H. The Dynamics and Thermodynamics of Compressible Fluid Flow. The Ronald Press Company, New York (1954).

[7] Starling K. E., Savidge J. L. Compressibility factors of natural gas and other related hydrocarbon gases. American Gas Association, Transmission Measurement Committee Report No. 8, Second Edition, Arlington, Virginia (1992), and Errata No. 1, (1994).

[8] Chekurin V.F., Khymko O. M. Mathemetical model for propagation of long acoustic waves in the flow of a gas pipeline. Proceedings of XXII International Seminar. Workshop on direct and inverse problems of electromagnetic and acoustic wave theory (DIPED). Dnipro, Ukraine (September 25-28, 2017), 80-83 (2017).

\title{
Математичне моделювання поширення малого збурення тиску в газовому потоці довгого трубопроводу
}

\author{
Чекурін В. ${ }^{1}$, Химко О. ${ }^{2}$ \\ ${ }^{1}$ Інститут прикладних проблем механіки і математики ім. Я. С. Підстригача НАН України \\ вул. Наукова, 3-б, 79060, Львів, Украӥна \\ ${ }^{2}$ Начіональний університет «Лввівсъка політехніка» \\ вул. С. Бандери, 12, 79013, Лъвів, Україна
}

Запропоновано математичну модель поширення малого збурення тиску, що виникло в потоці газу довгого трубопроводу. Модель містить два рівняння з частинними похідними, коефіцієнти яких виражаються через параметри початкового (незбуреного) потоку - густину маси і масовий потік. У разі стаціонарного незбуреного потоку коефіцієнти залежать лише від координати. У межах розробленої моделі чисельно досліджено вплив параметрів початкового стаціонарного процесу на поширення імпульсів збурення тиску і потоку, збуджених флуктуацією тиску, що виникла в потоці Розроблену модель можна застосувати для кількісного аналізу малих коливань режимів магістральних газопроводів, спричинених випадковими флуктуаціями потоку та/чи пульсаціями компресорів. Модель може бути корисною для дослідження поширення хвиль від'ємного перепаду тиску, які виникають у трубопроводі під час його локальної розгерметизації.

Ключові слова: нелінійні задачі, нестачіонарні прочеси, газова динаміка, газовий потік у довгому трубопроводі, хвилі у газі, що рухається, поширення малих збурень.

2000 MSC: 35Q35, 65M06

Удк: $534.13,519.6$

Mathematical Modeling and Computing, Vol.4, No. 2, pp. 126-138 (2017) 